\title{
Antonio Becchi, Hélène Rousteau-Chambon et Joël Sakarovitch (dir.), Philippe de La Hire (1640-1718). Entre architecture et sciences
}

Paris, Picard, 2013.

Robert Carvais

\section{OpenEdition}

Journals

Édition électronique

URL : https://journals.openedition.org/crau/459

DOI : $10.4000 /$ crau.459

ISSN : $2547-5746$

Éditeur

Éditions du patrimoine

Édition imprimée

Date de publication : 1 mars 2014

Pagination : 114-120

ISBN : 978-2-7577-0359-5

ISSN : 1296-4077

\section{Référence électronique}

Robert Carvais, «Antonio Becchi, Hélène Rousteau-Chambon et Joël Sakarovitch (dir.), Philippe de La Hire (1640-1718). Entre architecture et sciences », Les Cahiers de la recherche architecturale et urbaine [En ligne], 29 | 2014, mis en ligne le 13 septembre 2017, consulté le 21 septembre 2021. URL : http:// journals.openedition.org/crau/459; DOI : https://doi.org/10.4000/crau.459 


\section{Antonio Becchi, Hélène Rousteau-Chambon et Joël Sakarovitch (dir.) Philippe de La Hire (1640- 1718). Entre architecture et sciences}

Paris, Picard, 2013, 329 p., 39 euros.

L'intention des directeurs de cet ouvrage collectif et collaboratif, fruit d'un colloque international ${ }^{20}$, était d'étudier la vie et d'analyser l'œuvre de Philippe de La Hire, personnage énigmatique à plus d'un titre. À regarder les travaux qui lui ont été consacrés ${ }^{21}$, nous trouvons principalement des notices biographiques de dictionnaires généraux ou spécialisés, quelques renvois dans des ouvrages scientifiques et quelques rares articles. En revanche, la propre bibliographie de cet auteur couvre près de dix pages dans l'ouvrage ${ }^{22}$ entre ses travaux imprimés ou manuscrits, ses traités de cours, ses notices académiques sous les formes des plus variées de réflexions, remarques, observations, explications, descriptions, mémoires, exposés, études, etc. Le contraste apparaît trop important pour ne pas initier une telle recherche, d'autant que ses fonctions académiques sont multiples: membre de l'Académie royale des sciences dès 1678 (il en deviendra sous-directeur, voire directeur), professeur au Collège royal et astronome attaché à l'Observatoire de Paris dès 1682, professeur à l'Académie royale d'architecture dès 1687. Mais, au cœur de

20. Organisé à Paris du 24 au 26 juin 2010, par le laboratoire Géométrie-StructureArchitecture (GSA) de l'Énsa Paris-Malaquais, le département d'histoire et d'archéologie de l'université de Nantes et le Max-PlanckInstitut für Wissenschaftsgeschichte de Berlin, représentés par les trois directeurs de publication.

21. Voir les pages 310-311 de l'ouvrage.

22. Se référer aux pages 297-309. 
cet ouvrage se trouve la recherche unique chez tous les participants de retrouver pas à pas ce qui constitue le savoir essentiel de l'architecte moderne dans le foisonnement des sciences. Et l'on sera surpris $d^{\prime} y$ lire bien de lumineuses découvertes inédites, comme d'y constater des propositions de réponses à des interrogations posées aujourd'hui dans le champ de l'enseignement de l'architecture.

Joël Sakarovitch souligne dans un article introductif les deux intérêts à étudier Philippe de La Hire: d'une part ses capacités à travailler dans des domaines scientifiques des plus variés: cartographie, géométrie, botanique, hydraulique, gnomonique, mécanique, astronomie, physiologie, architecture, coupe des pierres: "une académie à lui tout seul», selon l'expression de Fontenelle dans l'éloge qu'il publia au décès de celui-ci (reproduite en annexe। de l'ouvrage). D'autre part, à travers ses prises de positions scientifiques, qu'elles fussent théoriques ou pratiques en réponse à une requête royale, Philippe de La Hire fait figure de "passeur entre deux mondes", en ce $\mathrm{XVII}$ e siècle qui voit la transformation de la société des Anciens en celle des Modernes. Au cœur de l'effervescence de l'activité scientifique européenne, dans la suite des travaux des Desargues, Roberval, Frénicle, concurremment à ceux de Newton, Leibniz, Varignon, Bernoulli, Huygens, Cassini, Picard, Philippe de La Hire a le mérite par la simplicité de ses écrits de former le point de départ de la nouvelle science. Il se positionne sans le savoir comme un " homme de transition ". Ainsi l'ouvrage se divise tout naturellement en deux parties: la première analyse la complexité de l'homme touche-àtout dans ses habits multiples lors de son voyage à Rome, selon ses fonctions occupées à l'Académie des sciences, à l'Observatoire ainsi qu'à l'Académie d'architecture et dans sa fidèle amitié avec son collègue Jean Picard. On aurait pu y joindre la convaincante analyse de son portrait présumé en savant (attribué à Gabriel Revel, ca. 1683) écrite par Thierry Verdier et rejetée à la fin de l'ouvrage. La comparaison des pages du livre représenté par le peintre avec le contenu des travaux géométriques de La Hire permet de reconnaître ce dernier avec une quasicertitude. La seconde partie, quant à elle, est réservée à l'étude de son œuvre qui, replacée dans un contexte savant très riche, souligne la convergence de ses pratiques scientifiques vers de nouveaux fondements de l'architecture.

\section{Les multiples facettes de l'homme de science ou les réseaux intellectuels de La Hire}

Thierry Verdier replace avec subtilité Philippe de La Hire dans le cénacle des savants lors de son voyage initiatique à Rome entre 1660 et 1664, malgré le peu de preuves qui nous soient parvenues. Arrivé comme peintre, il en repart conquis par l'esprit scientifique. Il est intéressant de noter que sa mère le pousse à la géomé- trie et lui ordonne de rentrer en France ${ }^{23}$. II s'intéresse à la création d'un cadran solaire réalisé pour le couvent de La Trinité-desMonts par les pères minimes, à un projet d'escalier monumental pour ce même couvent qui avait suscité des propositions du Bernin, de François d'Orbay ou d'Elpidio Benedetti. Mais il fréquente surtout la «nation » des artistes français, pour la plupart architectes "constructeurs» ou peintres qui n'omettent pas d'échanger avec le collège romain constitué d'intellectuels isolés, de savants enseignant dans les grandes congrégations religieuses réputées comme les Chartreux ou surtout les Jésuites.

Annie Chassagne analyse les 40 années d'activité de La Hire à l'Académie des sciences. Replaçant sa nomination dans son contexte politique, elle détaille le réseau scientifique acquis à sa cause. À l'origine, il apparaît comme mathématicien plutôt que comme physicien et consacre ses premiers travaux à l'astronomie mais aussi à diverses autres sciences. Bien sûr il répond aux ordres du roi concernant I'hydraulique de Versailles et à ceux de l'Académie désirant publier les ouvrages inédits de ses savants. À l'extrême fin du siècle, l'institution subit une profonde transformation qui interagit sur son travail: les travaux collectifs sont abandonnés au profit d'activités individuelles. Philippe de La Hire devient l'astronome de I'Académie et reste très actif. Le nombre de ses mémoires connaît une moyenne de sept à huit par an contre trois à quatre

23. Information donnée plus loin par Jean-Pierre Le Goff. 
pour ses collègues. II devient expert en mécanique, participe au fonctionnement de l'institution et accède ainsi aux plus hautes responsabilités académiques. L'auteur décrit plutôt un être solitaire n'ayant jamais profité des avantages de la cour, ne s'étant jamais conformé à l'idéal de la République des lettres. Assidu, dévoué et polyvalent, il demeure un savant au service de la science et du roi.

Guy Picolet, dans un long article passionnant et exemplaire à plus d'un titre, remarquablement bien documenté, retrace les relations savantes et énigmatiques entre Philippe de La Hire et son ami, "maître » et collègue Jean Picard durant leur vie et au-delà, après la mort de ce dernier. Grâce à une analyse fine des sources, l'auteur dresse un parallèle entre les deux hommes: socialement opposés, ils se retrouvent sur le plan intellectuel par leur approche empirique de la géométrie pratique via l'astronomie, la cartographie des côtes de la France, l'optique et la gnomonique. Héritier des travaux de son aîné, La Hire adapte son œuvre, en publie une grande partie et devient son suiveur. L'auteur, ayant renvoyé d'emblée la question du début de leur relation à la fin de sa démonstration, parvient habilement à nous convaincre de l'emprise de Picard sur La Hire au point d'en faire son "maître » à penser.

Jean Souchay traque le travail de Philippe de La Hire à I'Observatoire de Paris. Bien que commencé dès 1678 près de la porte Montmartre, les observations du héros sont régulières et fiables. Grâce au quart de cercle mural de Jean Picard, il mesure précisément la position des astres, des constellations, les thèmes astronomiques particuliers, les éclipses de lune et de soleil, voire les phénomènes exceptionnels. Ses tables de relevé sont publiées et servent souvent de sources par la suite, bien que Philippe de La Hire ait déjà conscience de ne pas disposer des outils newtoniens et des équations différentielles pour résoudre avec finesse le mouvement de notre satellite. Son analyse des plus de 8000 taches solaires a été vérifiée et corroborée de nos jours. De plus, le suivi régulier de la pluviométrie comme de la barométrie sert les intentions royales de remplir les réservoirs de Versailles. Cette surveillance du ciel donne l'occasion à La Hire de démontrer ses talents de "géomètres de l'espace " mais aussi de réaliser des expériences dans des domaines les plus éclectiques.

Hélène Rousteau-Chambon s'intéresse à Philippe de La Hire comme professeur à l'Académie royale d'architecture, dont son prédécesseur François Blondel est aussi homme de sciences. La Hire entretient non seulement un lien entre les deux académies mais développe un enseignement scientifique pour les deux publics. Déjà à l'Académie des sciences, nous relevons des travaux en rapport direct avec l'architecture. À l'Académie d'architecture ces champs d'intervention s'élargissent. Si les thèmes abordés devant les deux académies sont souvent identiques, les contenus en sont différents tant dans la forme que dans l'argumentaire. Le professeur adapte son discours en fonction du public auquel il s'adresse, souvent d'abord devant les architectes auxquels il expose ses inventions scientifiques, puis devant les scientifiques auxquels il explique l'architecture. II apporte ainsi un plus grand rayonnement à l'architecture savante. Comme professeur pour les futurs architectes du roi, il diversifie ses cours de géométrie appliquée à l'architecture aux principes, mais aussi à l'optique, la mécanique, l'hydrostatique, ou encore la perspective. II vise ainsi les ingénieurs. Et pour ces derniers comme pour les architectes, il délivre des cours d'hydraulique et $d^{\prime}$ architecture pour lesquels pragmatisme et expérimentation demeurent les maîtres mots, et inscrit par conséquent l'architecture au cœur de la science.

\section{Les œuvres de Philippe de La Hire au cœur de la science architecturale moderne}

Werner Oechslin ${ }^{24}$ introduit cette partie en démontrant comment Philippe de La Hire parvient à rejoindre l'architecture par le biais des mathématiques traditionnelles et précisément de la géométrie. Au fur et à mesure que l'édification passe au premier plan, les sciences deviennent indispensables à l'architecte. Déjà Vitruve inclut l'ingéniosité de l'architecte (capacité de démonter et d'expliquer) dans la ratiocinatio. La sciencia vitruvienne évolue en une discipline cartésienne. En effet,

24. On pourra lire une version plus complète de cet article en allemand sous le titre « Ratio und Vorstellungsvermögen, Geometrie! Philippe de La Hire, die wissenschaftliche Grunlage des Architektur in ihrer Ausrichtung auf die Praxis ", Scholion, Bulletin 7/2012, p. 73-132. 
toute l'ingéniosité de l'ingénieur est de rendre visible les figures, d'où la nécessité impérieuse de la géométrie pour l'imagination dans le recours aux dessins. Pour Descartes, cette science est une œuvre épistémologique décisive, une méthode simple et facile. L'enseignement scientifique de La Hire s'inscrit dans cette longue tradition de réflexion entre ratio et architecture, héritée de ses amis Descartes, Desargues et Bosse. La géométrie se trouve au cœur de leurs œuvres car ils veulent étendre les outils d'Euclide et d'Apollonius, perfectionner la représentation graphique et la mettre à la disposition de la mécanique et de la technique. Elle gère la maîtrise des questions constructives. Desargues n'écrit-il pas en 1647 que les maçons vont à l'école des géomètres et non l'inverse? Descartes lui-même réduit la géométrie à la pratique, comme Pierre Bullet, I'architecture au toisé. La géométrie permet de saisir les corps et leur représentation à travers des figures simples. La Hire I'utilise dès le début de ses travaux d'astronomie. Cependant il se montre réticent à l'égard du calculus car il appartient à la génération précédente de celle des Leibniz et Newton et car cette nouvelle préoccupation est avant tout théorique, contrairement aux besoins de I'Académie et de l'architecture. La question de la prééminence des Modernes sur les Anciens ne serait qu'un faux problème: le passage d'un monde à un autre sans pour autant dire que l'un est meilleur que I'autre. Le mérite de La Hire est d'avoir orienté la question des méthodes géométriques vers les besoins de l'architecture et d'avoir introduit la mécanique comme science calculable.

Jean-Pierre Le Goff en vient à considérer savamment les différents travaux de La Hire sur les coniques tout en les reliant à leur usage en architecture de son époque. Son étude chronologique et méticuleuse permet de démontrer en quoi La Hire emprunte dans ce domaine à ses contemporains et amis. II prouve en effet que son ignorance affichée de certaines publications antérieures sur le sujet semble mensongère. L'auteur montre également en quoi le héros a pu influencer certaines théories de Newton. Même s'il n'utilise le calcul infinitésimal qu'en dernier recours, il aurait voulu exposer que les Modernes (cartésiens) avaient surpassé les Anciens dans leurs méthodes pré-projectives. On pourra seulement regretter ici que l'auteur estime que le recensement des travaux de La Hire à l'Académie reste à faire alors que celui-ci a été admirablement réalisé par Guy Picolet dans l'annexe iv de l'ouvrage. Enfin, dans le passage sur le mémoire de 1702 (Remarques sur la forme de quelques Arcs dont on se sert dans l'Architecture), il est regrettable que La Hire soit maladroitement présenté comme un architecte qu'il n'a jamais été. Eut-il fallu préciser qu'il écrivait comme s'il avait été architecte!

Patricia Radelet présente ensuite le « traité de mécanique » (1695) de Philippe de La Hire dans le contexte des Principia de
Newton (1687) et des travaux de Varignon (son traité de 1687 et son «projet de nouvelle mécanique » de 1695). Alors que Newton étudie le mouvement grâce au calcul infinitésimal et intégral, La Hire traite de la statique et de son influence sur l'architecture qu'il enseigne à l'Académie depuis 1687. L'auteure dévoile ce qu'il doit à Borelli (De motu animalium, 1680, 1694, 1710). Cependant La Hire n'oublie pas de travailler sur le mouvement et par conséquent avec les infiniment petits et le calcul intégral. Mais s'il présente la vieille loi du levier, il est troublé par les travaux de Varignon qui promeut la loi des parallélogrammes des forces que La Hire redécouvre. L'auteure analyse enfin les deux propositions de La Hire qui sont remarquées par Jean Bernoulli en 1698 (CXXIII et cxxV) comme étant une découverte sans explication à défaut de l'usage du "calcul Leibnizien ». C'est ainsi que l'équilibre des voûtes demeure un enjeu principal dans le cadre de son enseignement à travers les questions de la caténaire et de la chaînette.

Antonio Becchi analyse, avec astuce et finesse, les allers et retours entre les manuscrits et les publications de Philippe de La Hire sur la question de la mécanique des voûtes. L'auteur remarque que même si La Hire se réfère à l'Antiquité, il ne cite que peu d'auteurs dont il s'inspire à l'exception d'Archimède, de Galilée et de ses amis contemporains comme Desargues. L'auteur montre bien que La Hire s'intéresse à la mécanique uniquement grâce 
à la géométrie pour laquelle l'architecture serait un cadre parfait d'application. En effet, quelle règle garantit la solidité des voûtes, archétype constructif? Passant par la lecture d'Alberti et de Derand, le piédroit doit avoir une largeur égale au quart du diamètre de la voûte. Cependant pour arriver à cette fin, il utilise la méthode artisanale " monolithique » qui idéalement projette l'ensemble de l'appareil comme s'il n'était qu'une seule et même pierre, l'objectif étant de définir une structure sans poussée latérale ou avec une poussée très limitée. Les écrits et manuscrits de l'Académie ${ }^{25}$ nous montrent l'évolution de La Hire sur la question. Insatisfait de sa première proposition, il suggère en 1712 une nouvelle règle générale afin de déterminer la largeur de la face des piédroits des arcs, c'est-à-dire l'effort de poussée sur la hauteur donnée des piédroits, ce qui manquait à Derand. Et cela grâce à deux machines simples pour examiner le point de rupture: le coin et le levier.

Luc Tamborero présente l'originalité du "traité de la coupe des pierres» de Philippe de La Hire. II montre comment il généralise la production antérieure sur un tel sujet, de De L'Orme à Derand, en passant par Jousse, Desargues et de Beaune, avec sa doctrine de l'angle solide. L'auteur retrace l'histoire des quatre manuscrits retrouvés de ce cours et qualifie de maître celui des Ponts et Chaussées, le plus complet en deux tomes (1688-1690), celui de la bibliothèque de I'Institut ayant servi de brouillon. La Hire reprend indé- niablement Desargues en le simplifiant. La surprise vient du second tome qui consiste en un cahier de travaux pratiques pour élèves. Ce fait est étayé par la correspondance qui figure dans le manuscrit et les propos d'autres de ses élèves des plus réputés, comme Frézier ou Lambert. Les épures préparées par ceux-ci sont intégrées au propre par La Hire dans son traité. Ainsi ce cours n'aurait pas entièrement été dicté comme habituellement à I'Académie, mais aussi «enrichi par ses élèves", comme si ceux-ci réalisaient leur chef-d'œuvre. De plus le texte de La Hire est d'une telle précision, usant un grand nombre de symboles, qu'il peut se passer du tracé. Enfin, il coupe le trait lui-même sans faire appel à un tailleur de pierre. Sans l'Académie royale d'architecture, les architectes n'auraient sans doute pas concurrencé le monopole des maçons protégés par la Chambre royale des bâtiments.

Hélène Rousteau-Chambon étudie le " cours d'architecture " de Philippe de La Hire qui nous est parvenu sous forme de deux manuscrits. Bien que n'étant pas architecte, La Hire connaît l'architecture, ses objets et ses auteurs. Par le biais des mathématiques et plus précisément de la géométrie, il maîtrise cette science au point de proposer de traduire un des auteurs les plus complexes, tel Vicenzo Scamozzi ${ }^{26}$. Son traité doit être considéré comme « un manuel d'architecture général », encyclopédique, aussi bien théorique que pratique. Alors que la théorie occupe le devant de la scène, il essaie d'être aussi pragmatique que le furent De L'Orme, Savot ou Bullet. Son cours est néanmoins atypique, la forme de son texte innovant et son discours inhabituel. Archaïque, il ne présente pas la comparaison des proportions des éléments de chaque ordre promue par Serlio et Fréart. Fougueux, il n'hésite pas à polémiquer avec ses collègues Desgodets et Bullet, à propos de l'attique qu'il réprouve sans toutefois incriminer directement le surintendant à ce sujet. II prône avec beaucoup de rigueur le dessin qu'il connaît par son père et insiste sur le fait que l'architecte doit avoir du recul par rapport à son projet, usant ainsi d'autocritique.

Madeleine Pinault Sørensen décrit ensuite le "traité de la pratique de la peinture" de La Hire. Elle rappelle fort heureusement qu'il avait contribué à " un art de la peinture " dans la Description des arts et métiers sous la direction de l'abbé Bignon, publié tardivement. Son traité de la pratique de la peinture, lui, sera publié posthume, mais plus tôt, en 1730 . Ses sources, en dehors de sa pratique personnelle, se trouvent principalement dans l'écrit que consacre André Félibien à cet art publié en 1676. II s'inspire du plan et du contenu, sauf que La Hire ne cite aucun artiste, ni œuvre particulière, à l'exception de Bernard Palissy qui pourtant est encore en disgrâce à l'époque. L'auteure s'interroge pour savoir si référence est faite au peintre ou au protestant banni. La Hire rédige méthodiquement un traité pratique, sur les données techniques
25. L'auteur précise que certains mémoires n'ont pas été publiés par Henry Lemonnier lors de la publication des procès-verbaux de l'Académie au début du xxe siècle, ce qui plaiderait en faveur d'une révision de cette édition.
26. Voir à ce sujet Olga Medvedkova, "Scamozzi en français: histoire d'un échec », dans Robert Carvais (dir.) et al., Traduire l'architecture, à paraître. 
et les outils utilisés pour peindre. Son regard d'excellent dessinateur se penche d'abord sur le dessin dont il livre les outils et méthodes, puis aux sept techniques de la peinture.

\section{La naissance de la science constructive: du savoir-faire à la compétence}

Pascal Dubourg Glatigny termine I'ouvrage en s'interrogeant sur les fondements académiques de la science de la construction. Cette question constitue en réalité le ciment de cet ouvrage collectif. D'autres personnages que La Hire, comme Claude Perrault et François Blondel, ont déjà situé leur parcours entre science et architecture. Quelle est la particularité de La Hire sur ce point? Avec ce personnage nous nous trouvons face à une figure intellectuelle en pleine contradiction. Comment un nonpraticien s'intéresse-t-il à la technique architecturale? Le statut de l'architecture comme discipline professionnelle au cours du XVIII ${ }^{e}$ siècle permet alors de dresser trois postulats afin de mieux comprendre ce paradoxe:

- Écrire sur l'architecture n'est pas réservé uniquement aux architectes, et cela serait valable pour toutes les sciences. La preuve en est que les premiers à écrire et à appliquer le droit en matière de construction ne sont rien d'autres que des maçons et / ou des architectes ${ }^{27}$.

- Enseigner une discipline n'oblige pas à l'exercer ni même à l'avoir un jour pratiquée. Si La Hire est professeur d'architec- ture, il n'a pas enseigné le projet mais les outils permettant aux élèves une réflexion aussi bien sur la conception que sur le chantier, sans pour autant que cet enseignement soit pratique, mais concerne la théorie d'une pratique. Les enseignants de projet aujourd'hui dans les écoles d'architecture n'ont parfois jamais pratiqué I'architecture. Est-ce pour autant acceptable? Avec La Hire nous assistons au passage du savoir-faire à la compétence. II professe un savoir qu'il maîtrise mais qu'il n'exerce pas.

- La théorie peut ne pas être si éloignée de la pratique. L'opposition vitruvienne classique entre les deux concepts de ratiocinatio et de fabrica mériterait d'être alors remise en cause et en œuvre. Ainsi il n'est pas si bizarre de penser une théorie de la pratique comme avec La Hire. La transmission du savoir selon l'auteur s'opère dans l'interface entre le processus «intellectuel » et la « procédure matérielle ».

Finalement Philippe de La Hire emprunte une part différente aux deux figures fondamentales du moment. II est institutionnel comme François Blondel, à l'inverse de Claude Perrault. II n'exerce pas, presque comme Perrault, à la différence de Blondel. Il s'en différencie aussi car moins organique et moins politique. La Hire n'apparaît ni totalement homme universel, ni totalement humaniste, ni totalement néovitruvien. Il est une des dernières figures maîtrisant à la fois les sciences de l'ingénieur et celles de l'architecture. Ce ne sera plus valable ni chez Newton, ni chez Leibniz. La Hire adopterait la posture sans qu'il l'ait choisie d'un Giovanni Poleni, précurseur du néovitruvianisme. L'auteur émet I'hypothèse selon laquelle La Hire ne serait pas à cheval entre deux mondes ancien et nouveau mais une figure exemplaire prenant une posture particulière à un moment de la redéfinition des champs disciplinaires, parvenant à «théoriser des pratiques normatives par le moyen de la géométrie ». II serait le premier à poser les fondations d'un art de bâtir ancré à une science de la construction qui se peaufinera tout au long du xvIII siècle chez ses successeurs (de Desgodets à Blondel) pour aboutir finalement au cœur de I'œuvre de Rondelet.

Cet ouvrage constitue donc une réussite sur deux plans: d'abord, en apportant un éclairage neuf sur un personnage et une œuvre clé pour comprendre la constitution de l'architecture moderne et ensuite, en ouvrant d'inévitables pistes de recherches. En effet, une de ses particularités est sa modestie et les ouvertures auxquelles elle invite le lecteur.

Annie Chassage évoque les conflits entre La Hire et ses collègues qui pourtant ne filtrent pas en dehors de l'institution. II apparaît difficile d'apprécier sa position lors de controverses savantes. Est-il resté neutre? A-t-il pris position en faveur des uns ou des autres? La question reste ouverte faute de sources claires sur la question. La véritable posture adoptée par La Hire face à la situation scientifique de son temps, en particulier entre les Anciens
27. Nous renvoyons sur ce point à nos travaux. Voir par exemple, "La littérature juridique du bâtiment. L'invention et le succès d'un genre doublement technique. 1748-1950 » dans Jean-Philippe Garric, Valérie Nègre, Alice Thomine-Berrada (dir.), La Construction savante. Les avatars de la littérature technique, Paris, Picard, 2008, p. 89-102; «Quand les architectes jugeaient leurs pairs, les juristes représentaient-ils encore le droit des bâtiments? L'histoire des relations pratiques entre droit et architecture ", Droit et Ville, $n^{\circ} 76$, décembre 2013; " L'élaboration d'un nouveau champ juridique à travers ses manuels: Des loix des bastimens au droit de la construction » dans Anne-Sophie Chambost et al. (dir.), Des traités aux manuels de droit. Une histoire de la littérature juridique comme forme du discours universitaire, colloque de I'université Paris-Descartes, en collaboration avec le Centre de théorie et analyse du droit et l'École de droit de Sciences Po, Paris, Dalloz, à paraître. 
et les Modernes, demeure une énigme et partage les auteurs. Antonio Becchi oppose un La Hire, plutôt « ancien » usant de géométrie, face à un Varignon, plutôt moderne usant du calcul infinitésimal. Refusant de choisir un camp, Joël Sakarovitch penche en faveur d'une attitude de compromis, Werner Oechslin et Pascal Dubourg Glatigny refusent la dichotomie pour positionner La Hire dans une tout autre dimension.

Guy Picolet suggère par ailleurs de poursuivre l'étude des relations entre Picard et La Hire à partir de l'analyse de leur correspondance, pour déterminer le plus précisément possible la part d'influence de Picard sur La Hire. II resterait encore à comprendre les ressorts et les fonctionnements des membres de la compagnie, surtout avant et après la réforme de 1699.

Antonio Becchi souligne enfin d'autres questions méritant de nouveaux développements: la grande originalité du modèle mécanique de La Hire dans ses rapports mécanique / architecture, comme dans le fait d'avoir fait appel à la géométrie dans l'explication de la coupe des pierres; I'appel à la mécanique des animaux, selon une grande tradition antique de Borelli à Perrault dans laquelle le calcul ne sert à rien. Nous aurions aimé lire deux contributions supplémentaires à cet ouvrage: une sur les fils de Philippe de La Hire, Augustin (16881744), ingénieur des Ponts et Chaussées, Jean Nicolas (1685-1727), botaniste et surtout I'aîné, Gabriel Philippe (16771719) qui a occupé les mêmes fonctions

28. Dans une étude que nous menons actuellement avec Philippe Bernardi et Joël Sakarovitch sur «la controverse de Bédoin», si l'entrepreneur-architecte Pierre Thibault possède dans sa bibliothèque le traité de coupe de pierres resté manuscrit, son contradicteur l'ingénieur-architecte Antoine d'Alleman invoque, lui, directement le traité de mécanique de La Hire dans le mémoire de la dispute (1732). Par ailleurs, une copie du mémoire de La Hire sur les grosseurs des bois (Académie royale d'architecture, 1688) que son père mais sur un temps très court et avec lequel il a beaucoup travaillé; et une autre sur la diffusion de son œuvre tant chez les architectes français qu'à l'étranger ${ }^{28}$. Enfin nous ne saurions que trop inciter les éditeurs à envisager une édition numérique des travaux de La Hire, ou au moins ceux n'existant que sous forme manuscrite, permettant ainsi l'établissement de textes maîtres, la diffusion de l'œuvre dans son intégralité et des comparaisons fructueuses avec les bases scientifiques du savoir architectural passé, contemporain et futur ${ }^{29}$.

$\mathrm{Si}$ les outils biobibliographiques et les chronologies de l'ouvrage se révèlent fort utiles au lecteur, nous regretterons quelques répétitions entre les différentes contributions qui auraient pu être esquivées par des renvois mutuels. Enfin, l'éditeur aurait pu éviter de reproduire des documents dans le sens contraire de la lecture courante, en particulier pour les annexes.

Cet ouvrage marquera, sans nul doute, I'historiographie d'histoire de la construction et de l'architecture comme apportant une pierre neuve à l'édifice complexe de la naissance de la science constructive dans l'environnement architectural.

ROBERT CARVAIS

Directeur de recherche CNRS / UMR 7074, Centre de théorie et analyse du droit, équipe Théorhis.

se retrouve dans la collection des livres, des desseins et des estampes de Carl Hårleman, architecte, puis surintendant à la cour, à Stockholm (Bibliothèque royale, Manuscrit S33, pièce 12).

29. À l'instar de ce que nous avons entrepris pour les cours d'Antoine Desgodets à I'Académie dans le cadre d'un projet ANR, réalisé entre 2007 et 2013 [à paraître en ligne]. 